\title{
Automorphism groups of totally ordered sets: a retrospective survey.
}

\author{
V. V. Bludov, M. Droste and A. M. W. Glass
}

July 23, 2009

To Charles on his $75^{\text {th }}$ birthday with our gratitude for his research, help and friendship.

\begin{abstract}
In 1963, W. Charles Holland proved that every lattice-ordered group can be embedded in the lattice-ordered group of all orderpreserving permutations of a totally ordered set. In this article we examine the context and proof of this result and survey some of the many consequences of the ideas involved in this important theorem.
\end{abstract}

\section{Genesis}

In 1957, P. M. Cohn noted that Cayley's (right regular) Representation Theorem applies immediately to right-ordered groups [12]. That is, let $G$ be a group with a total order that is preserved by multiplication on the right. Embed $G$ in the group $A(G):=A u t(G, \leq)$ of all order-preserving permutations of the $\operatorname{set}(G, \leq)$ via the right regular representation; i.e.,

AMS Classification: 06F15, 20F60, 20B27, 20F10.

Keywords: totally ordered set, permutation group, representation, primitive permutation group, amalgamation, free product with amalgamated subgroup, right-orderable group, normal subgroups, Bergman property, outer automorphism groups. 
$f(g \vartheta)=f g \quad(f, g \in G)$. Now $A(G)$ can be right totally ordered as follows: let $\prec$ be any well-ordering of the set $G$ and define $a<b$ iff $\alpha_{0} a<\alpha_{0} b$, where $\alpha_{0}$ is the least element of $G$ (under $\prec$ ) of the support of $b a^{-1}$. If we choose $\prec$ so that its least element is the identity of $G$, then the right regular embedding preserves the group operation and the total order: $g_{1}<g_{2}$ in $G$ iff $g_{1} \vartheta<g_{2} \vartheta$ in $A(G)$.

What can one do if the order on $G$ is not total, even if multiplication on the left also preserves the partial order? The easiest situation would be to consider lattice-ordered groups. (We will use the abbreviation $\ell$ groups for these throughout.) That is, $G$ is a group and a lattice with

$$
a(f \vee g) b=a f b \vee a g b \quad \text { and } \quad a(f \wedge g) b=a f b \wedge a g b \quad(a, b, f, g \in G) .
$$

So if we write $f \leq g$ for $f \vee g=g$ (or, equivalently, $f \wedge g=f$ ), then we have $a f b \leq a g b$ whenever $f \leq g$. If the lattice order on an $\ell$-group $G$ is total ( $f \leq g$ or $g \leq f$ for all $f, g \in G$ ), then we say that $G$ is an o-group. The structure theory of abelian o-groups was explored at the beginning of the last century. It is rich and reasonably well understood. The study of the structure of abelian $\ell$-groups was begun in the 1930s from a functional analysis viewpoint, and more abstractly in the 1950s and 1960s by Paul F. Conrad and his students. Conrad began to study the structure of non-abelian $\ell$-groups around 1960 but results were limited. So a Cayley-type theorem could be very helpful. But there is a very big problem. Consider the very easy abelian $\ell$-group $G:=\mathbb{Z} \oplus \mathbb{Z}$, where

$$
\left(m_{1}, n_{1}\right) \vee\left(m_{2}, n_{2}\right):=\left(\max \left\{m_{1}, m_{2}\right\}, \max \left\{n_{1}, n_{2}\right\}\right) .
$$

If we consider the group of order-preserving permutations of this lattice, then the "switch in coordinates" $(m, n) \mapsto(n, m)$ belongs to $A(G)$ but composed with itself yields the identity. So $A(G)$ has an element of order 2 which cannot happen in any $\ell$-group ([31], Lemma 1.11.1). Is there any way to get round this obstacle? This was the problem facing Holland in 1962/63 when he was at the University of Tübingen with Helmut Wielandt.

In contrast to the lattice case, if $(\Omega, \leq)$ is any totally ordered set, then $A(\Omega):=A u t(\Omega, \leq)$ is an $\ell$-group under the pointwise ordering

$$
\alpha(f \vee g)=\max \{\alpha f, \alpha g\}, \quad \alpha(f \wedge g)=\min \{\alpha f, \alpha g\} \quad(\alpha \in \Omega ; f, g \in G) .
$$


Using this, Holland elegantly solved the problem. As was already well known, if $G$ is an $\ell$-group and $g \in G$ with $g \neq 1$, there is a convex sublattice subgroup $V_{g}$ of $G$ which is maximal with respect to not containing $g$ (use Zorn's Lemma). It is called a value of $g$ (it first appeared in the totally ordered abelian case in valuation theory). Holland used the fact that $\Omega_{g}$, the set of right cosets of $V_{g}$ in $G$, inherits a natural total order $\left(V_{g} f \leq V_{g} h\right.$ iff $v f \leq h$ for some $v \in V_{g}$ ). Now $G$ acts on $\Omega_{g}$ preserving order. This provides a group homomorphism of $G$ into $A\left(\Omega_{g}\right)$ that also preserves the lattice operations. It is faithful (and so does what we want) iff the only convex normal sublattice subgroup of $G$ contained in $V_{g}$ is the identity. If this is not the case, we need to modify the construction. Holland first took the full Cartesian product $C:=\prod\left\{A\left(\Omega_{g}\right) \mid g \in G \backslash\{1\}\right\}$. It inherits a lattice structure in the natural way (coordinatewise) and the natural map provides an $\ell$-embedding of $G$ into $C$ (if $f \neq 1$, then the $f$-coordinate of the image of $f$ moves $V_{f}$ and so is not the identity). But one can do better [39]. Let $\prec$ be any total order of $G \backslash\{1\}$ and totally order $\Omega:=\bigcup\left\{\Omega_{g} \mid g \in G \backslash\{1\}\right\}$ by: let $\alpha \in \Omega_{f}$ and $\beta \in \Omega_{g}$; then $\alpha<\beta$ iff either $f \prec g$ or $f=g$ and $\alpha<\beta$ in $V_{f}$. Furthermore, the natural embedding $\varphi: G \rightarrow A(\Omega)$ given by

$$
\left(V_{f} h\right)(g \varphi):=V_{f}(h g) \quad(f, h, g \in G)
$$

is an $\ell$-embedding (i.e., the group and lattice operations are preserved). Thus Holland established

Theorem A (Holland [39]) Every lattice-ordered group can be $\ell$-embedded in $A(\Omega)$ for some totally ordered set $(\Omega, \leq)$.

The proof of the pudding lies in its uses, some of which will be related in the next section. But an immediate one to whet the appetite was provided in the original paper. B. H. Neumann had asked: Can every orderable group be embedded in a divisible orderable group ? $^{1}$. Assuming the Generalised Continuum Hypothesis (G.C.H), Holland proved in [39] that every $\ell$-group can be $\ell$-embedded in a divisible $\ell$-group. We will sketch a proof of this in the next section avoiding G.C.H.

${ }^{1}$ A negative answer was finally provided this century by Vasily V. Bludov $[6]$. 


\section{Some applications}

\subsection{Divisibility}

Let $(\Omega, \leq)$ be a totally ordered set and $F:=F(\Omega)$ be the free abelian group on the set $\Omega$. The total ordering on $\Omega$ lifts to a total ordering on $F$ in the natural way: if $\alpha_{n_{0}}=\max \left\{\alpha_{i} \mid i=1, \ldots, n\right\}$ in $\Omega$, then $\sum_{i=1}^{n} m_{i} \alpha_{i}>0$ in $F$ iff $m_{n_{0}} \in \mathbb{Z}_{+}$. With this ordering, $F$ is an abelian o-group. The group ring $\mathbb{Q}[F]$ is an integral domain. By extending the ordering from $F$ to $\mathbb{Q}[F]$ in the natural way $\left(\sum_{f \in F} q_{f} f>0\right.$ iff $q_{f_{0}}>0$, where $\left.f_{0}=\max \left\{f \in F \mid q_{f} \neq 0\right\}\right), \mathbb{Q}[F]$ becomes an abelian o-group. If $\Lambda$ is the field of quotients of $\mathbb{Q}[F]$, then the order on $\mathbb{Q}[F]$ extends to a total order on $\Lambda$. It can be shown that $A(\Omega)$ can be $\ell$-embedded in $A(\Lambda)$ (see [67]). Thus E. C. Weinberg proved that every $\ell$-group can be $\ell$-embedded in an infinite o-2 transitive $\ell$-permutation group $A(\Lambda)$ (i.e., if $\alpha_{1}<\beta_{1}$ and $\alpha_{2}<\beta_{2}$, then there is $g \in A(\Lambda)$ such that $\alpha_{1} g=\alpha_{2}$ and $\beta_{1} g=\beta_{2}$ ) as, clearly, the group of order-preserving linear maps of any ordered field is o- 2 transitive. It is easy to see that infinite o- 2 transitive $\ell$-permutation groups are o- $n$ transitive for all $n \in \mathbb{Z}_{+}$. Hence if $f \in A:=A(\Lambda)$ and $m \in \mathbb{Z}_{+}$, then $f$ and $f^{m}$ are conjugate in $A$. Let $g \in A$ be such that $g^{-1} f g=f^{m}$. Then $\left(g f g^{-1}\right)^{m}=f$. So $A$ is a divisible $\ell$-group and the consequence mentioned at the end of the previous section follows. Putting $m=2$ we see that $f=[f, g]:=f^{-1} g^{-1} f g$. Thus every element of $A$ is a commutator and so $A$ is perfect (i.e., it equals its derived subgroup) if $A=A(\Lambda)$ is o-2 transitive. We will examine this phenomenon in a later Subsection.

For any given totally ordered set $(\Omega, \leq)$, we have constructed an ordered field $\Lambda$ containing $\Omega$ such that $A(\Omega) \ell$-embeds into $A(\Lambda)$. We can also find an ordered set $(\Lambda, \leq)$ such that $\Omega \subseteq \bar{\Lambda} \backslash \Lambda$ (where $\bar{\Lambda}$ denotes the Dedekind completion of $\Lambda), A(\Lambda)$ is o- 2 transitive, and $A(\Omega) \ell$-embeds into $A(\Lambda)$. For this, identify $\Omega$ with $\Omega \times\{1\} \subseteq \Omega \times\{1,2\}$, ordered lexicographically from the left. Then we insert copies of a suitable dense order into the 'gaps' between $(\alpha, 1)$ and $(\alpha, 2)$ in $\Omega \times\{1,2\}$ for each $\alpha \in \Omega$, and enlarge this chain to $\Lambda$ to make $A(\Lambda)$ o-2 transitive, cf. [21, 14]. Holland's method [39] shows that $A(\Omega)$-embeds into $A(\Lambda)$. This construction of $\Lambda$ can be performed so that the inclusion id $: \Omega \times\{1,2\} \rightarrow \bar{\Omega}$ preserves all existing suprema and infima. This will be useful later on. 


\subsection{Normal subroups of $A(\Omega)$}

In his 1963 paper, Holland also found all the convex normal sublattice subgroups of $A(\mathbb{R})$, where $\mathbb{R}$ is the real line with the usual ordering. Besides $\{1\}$ and $A(\mathbb{R})$, these are

$$
\begin{gathered}
B(\mathbb{R}):=\{g \in A(\mathbb{R}) \mid(\exists \alpha, \beta \in \mathbb{R})(\operatorname{supp}(g) \subseteq(\alpha, \beta))\}, \\
L(\mathbb{R}):=\{g \in A(\mathbb{R}) \mid(\exists \beta \in \mathbb{R})(\operatorname{supp}(g)<\beta)\}, \quad \text { and } \\
R(\mathbb{R}):=\{g \in A(\mathbb{R}) \mid(\exists \alpha \in \mathbb{R})(\operatorname{supp}(g)>\alpha)\},
\end{gathered}
$$

where, in general, $\operatorname{supp}(g):=\{\alpha \in \Omega \mid \alpha g \neq \alpha\}$. The proof is quite simple and pictorial. Given any $g \in B(\mathbb{R})$ with $g>1$ (i.e., $\tau g \geq \tau$ for all $\tau \in \mathbb{R}$ and $\tau_{0} g>\tau_{0}$ for some $\tau_{0} \in \mathbb{R}$ ), for any $f \in B(\mathbb{R})$ with $f \geq 1$, one can find $h \in B(\mathbb{R})$ such that $\tau_{0} h<\operatorname{supp}(f)<\tau_{0} g h$, where $\tau_{0}$ is an element of $\operatorname{supp}(g)$. Then for any $\sigma \in \operatorname{supp}(f)$, we have $\tau_{0} h<\sigma<\sigma f<$ $\tau_{0} g h<\sigma h^{-1} g h$. Hence $1 \leq f<h^{-1} g h$. More generally, if $a \in B(\mathbb{R}) \backslash\{1\}$, either $a \vee 1>1$ or $a^{-1} \vee 1>1$. Thus any convex normal subgroup of $B(\mathbb{R})$ other than $\{1\}$ must contain all strictly positive elements of $B(\mathbb{R})$. But in any $\ell$-group, $a=(a \vee 1)\left(a^{-1} \vee 1\right)^{-1}$. Hence $B(\mathbb{R})$ is $\ell$-simple (i.e., it has no non-trivial normal convex sublattice subgroups). (The same argument holds with $\mathbb{R}$ replaced by any totally ordered set $(\Omega, \leq)$ with $A(\Omega)$ o-2 transitive.) If $1<g \in L(\mathbb{R}) \backslash B(\mathbb{R})$, then for each $f \in L(\mathbb{R})$ with $f \geq 1$, one can similarly find $h_{1}, h_{2} \in L(\mathbb{R})$ such that $1 \leq f \leq h_{1}^{-1} g h_{1} \vee h_{2}^{-1} g h_{2}$. This suffices to show that the only non-trivial normal convex sublattice subgroup of $L(\mathbb{R})$ is $B(\mathbb{R})$. Mutatis mutandis $R(\mathbb{R})$; and the result for $A(\mathbb{R})$ follows since each element of $A(\mathbb{R})$ greater or equal to 1 can be written in the form $f_{1} \vee f_{2}$ where $f_{1} \in L(\mathbb{R})$ and $f_{2} \in R(\mathbb{R})$. Actually, $B(\Omega)$ is a simple group if $A(\Omega)$ is o-2 transitive, as was shown earlier by Graham Higman $[37]^{2}$. However, if we replace $\mathbb{R}$ by $(\Omega, \leq)$ where $A(\Omega)$ is o-2 transitive, the normal subgroup picture can be far more complicated.

\footnotetext{
2 So, by Weinberg's result, every $\ell$-group can be $\ell$-embedded in an $\ell$-group that is simple as a group.
} 
First, if $\Omega$ contains a countable subset without an upper or lower bound in $\Omega(e . g ., \mathbb{Z}$ in $\mathbb{R}$ or in $\mathbb{Q})$, then $B(\Omega), L(\Omega)$, and $R(\Omega)$ constitute all proper non-trivial normal subgroups of $A(\Omega)$. But if each countable subset of $\Omega$ has an upper or lower bound in $\Omega$, then $A(\Omega)$ contains chains of normal subgroups isomorphic to $w_{1}$, the first uncountable ordinal, or to $(\mathbb{R}, \leq)$, and $A(\Omega)$ has at least $2^{2^{\aleph_{1}}}$ maximal proper normal subgroups. Many further properties of the normal subgroup lattice are given in [3, 13]. In spite of their apparent complexity, a set-theoretic construction of all normal subgroup lattices of o- 2 transitive groups $A(\Omega)$ was given in [21]. This rests on the construction method of orders $\Lambda$ described at the end of Subsection 2.1.

We note that the situation also changes if $(\Omega, \leq)$ is only assumed to be semilinearly (instead of totally) ordered. Let us call a partially ordered set $(T, \leq)$ a tree, if any two elements have a common lower bound but no two incomparable elements have a common upper bound, and it contains an infinite chain and at least two incomparable elements. Such trees and their automorphism groups occurred, e.g., in a classification theorem of certain Jordan groups [2]. There are $2^{\aleph_{0}}$ pairwise non-isomorphic countable trees $(T, \leq)$ with $A(T)$ o-2 transitive [19]. Using Holland's method [39] for describing conjugacy of elements, it was shown in [18] that if $T$ is countable, then $A(T)$ contains a smallest non-trivial and a largest proper normal subgroup, but there is an antichain of normal subgroups of size $2^{2^{\aleph_{0}}}$ between them.

\section{3 o-Primitive $\ell$-permutation groups.}

If $G$ is an $\ell$-group $\ell$-isomorphic to a sublattice subgroup of $A(\Omega)$, we say that $(G, \Omega)$ is an $\ell$-permutation group. If $(G, \Omega)$ is an o- 2 transitive $\ell$-permutation group, then it is o-primitive: the only $G$-congruences on $\Omega$ with all equivalence classes convex are the obvious two:

$$
\alpha \mathcal{E} \beta \quad \text { iff } \quad \alpha=\beta, \quad \text { and } \quad \alpha \mathcal{U} \beta \quad \text { for all } \alpha, \beta \in \Omega \text {. }
$$

Primitive permutation groups are the building blocks in the study of general permutation groups, so the next development had to be the classification of all o-primitive automorphism groups $A(\Omega)$. In 1965, Holland showed that these are either o- 2 transitive or $(\Omega, \leq)$ is isomorphic to a 
subgroup of $\mathbb{R}$ and the action of $A(\Omega)$ is just the right regular representation [40]; so $A(\Omega)$ is just an archimedean (abelian) o-group in the latter case. In [61], Ohkuma demonstrated that there are $2^{2^{\aleph_{0}}}$ of these subgroups $H$ of $\mathbb{R}$ with $A(H) \cong H$, the maximum possible number.

What about the more general situation when $G$ is a sublattice subgroup of $A(\Omega)$ for some totally ordered set $(\Omega, \leq)$ ? Can one obtain something akin to the O'Nan-Scott Theorem or the results of Adeleke and P. M. Neumann [2] and others?

Consider

$$
G:=\{g \in A(\mathbb{R}) \mid(\forall \alpha \in \mathbb{R})((\alpha+1) g=\alpha g+1)\} .
$$

It is easy to see that $(G, \mathbb{R})$ is transitive and o-primitive. It is not o-2 transitive as there is no element of $G$ mapping 0 to 0 and 1 to 2. Also, since any order-preserving permutation of $[0,1]$ extends to an element of $G$, we see that $G$ is o- 2 transitive on $(0,1)$ - and so on $(\alpha, \alpha+1)$ for any $\alpha \in \mathbb{R}$ - and not totally ordered. Note that the element $z: \alpha \mapsto \alpha+1$ is in the centre of $G$ and $G$ is the centraliser of $z$ in $A(\mathbb{R})$. We call $z$ the period of $G$. More generally, let $(G, \Omega)$ be an $\ell$-permutation group and $(\bar{\Omega}, \leq)$ be the Dedekind completion of $(\Omega, \leq)$. Suppose that $z \in A(\bar{\Omega})$ has the property that for any $\alpha, \beta \in \Omega$ there are $m, n \in \mathbb{Z}$ with $m<n$ such that $\alpha z^{m}<\beta<\alpha z^{n}$ and $G=C_{A(\Omega)}(z)$, the centraliser of $z$ in $A(\Omega)$. Then we say that $(G, \Omega)$ is periodic with period $z$.

In his 1967 Ph.D. Thesis under Holland, S. H. McCleary proved:

Theorem B (The Trichotomy Theorem [54]) Let $(G, \Omega)$ be a transitive, o-primitive $\ell$-permutation group. Then either

(i) $(G, \Omega)$ is o-2 transitive,

(ii) $(\Omega, \leq)$ is isomorphic to a subgroup of $\mathbb{R}$ and $(G, \Omega)$ is the right regular representation $(G \cong \Omega$ as o-groups $)$, or

(iii) $(G, \Omega)$ is periodic with period $z$ for some $z \in A(\bar{\Omega})$. In this case, for any $\alpha \in \Omega, G$ is o-2 transitive on $(\alpha, \alpha z) \cap \Omega$.

In 1976, McCleary [55] obtained a similar result without the transitivity assumption.

Having classified the o-primitive (transitive) $\ell$-permutation groups, the next task was to determine how these o-primitive "components" could 
be sewn together. Even in the standard permutation group setting, this was not easy to achieve. In 1969, Holland constructed a generalised Wreath product of permuation groups [41] and, with McCleary, obtained a similar result for $\ell$-permutation groups [45]. This was used by J. A. Read [64] in the special case that the $\ell$-group has a plenary set of values normal in their covers. Indeed, $(G, \Omega)$ is a normal-valued $\ell$-group if and only if $(G, \Omega)$ has non-overlapping intervals of support (if $f, g \in G$ and $\alpha, \beta \in \Omega$, then the convexifications of the interval of support of $\alpha$ under $f$ and $\beta$ under $g$ are either disjoint or one contains the other). In this case, $(G, \Omega)$ can be $\ell$-embedded in a subdirect product of $\ell$-permutation groups each of which has the form $\operatorname{Wr}\{(\mathbb{R}, \mathbb{R}) \mid \gamma \in \Gamma\}$ for some totally ordered set $\Gamma$, the action $(\mathbb{R}, \mathbb{R})$ being the right regular one (see [31] Chapter 11, [45], [64] and [32] Chapter 4 for precise definitions). This is very closely related to the work of Navas and Rivas [60] who prove that a right-ordered group $G$ is Conrad right-ordered if and only if it has a permutation representation which is non-overlapping ("non-crossing" in their terminology).

\subsection{Varieties, Free $\ell$-Groups, and Free Products of $\ell$-Groups}

Holland next applied the Trichotomy Theorem to the study of varieties of $\ell$-groups. In 1976 he showed that

Theorem $\mathbf{C}$ Let $(G, \Omega)$ be an o-primitive $\ell$-permutation group that is either o-2 transitive or periodic. Then the variety of $\ell$-groups defined by $G$ is the variety of all $\ell$-groups.

Hence, by Read's result and the Trichotomy Theorem, the class of all normal-valued $\ell$-groups (which S. Wolfenstein proved is a variety [69]) is the largest proper variety of $\ell$-groups. In fact, it is generated by the class of all $\ell$-soluble $\ell$-groups - see [34] where the structure of the lattice semigroup of varieties of $\ell$-groups was fully investigated using the Holland representation (Theorem A).

Permutation ideas can also be used to prove that any free $\ell$-group on a finite $(\geq 2)$ or countably infinite set of generators is $\ell$-isomorphic to an o-2 transitive sublattice subgroup of $A(\mathbb{Q})$ (see [26], [56], and [49]). 
The proof can be extended to the free product (in the class of $\ell$ groups) of any finite set of non-trivial countable $\ell$-groups [27]. The ideas of [43] were also used to show that any free $\ell$-group has soluble word problem [46], thus starting the study of decision problems in $\ell$-groups. We sketch an outline of the proof which again involves the ideas of [39].

Let $F$ be a free $\ell$-group on a finite number of generators. We may regard $(F, \mathbb{Q})$ as an $\ell$-permutation group. Let $w$ be a group word; say $w:=x_{1} x_{2}^{-1} x_{1}^{2}$. Draw two diagrams, one with $0 x_{1}>0$, the other with $0 x_{1}<0$. Now $x_{2}$ is a different generator from $x_{1}$ so, in the first diagram, we can put $0 x_{1} x_{2}^{-1}>0 x_{1}$ or $0 x_{1}>0 x_{1} x_{2}^{-1}>0$ or $0 x_{1} x_{2}^{-1}<0$, thus extending the first diagram in three possible ways. Similarly, we get three possible diagrams in the second case, making a total of six. Let's consider, for example, the diagram $0 x_{1}>0 x_{1} x_{2}^{-1}>0$. Now $x_{1}$ is orderpreserving, so we must have $0 x_{1} x_{2}^{-1} x_{1}>0 x_{1}$ and, by the same argument, that $0 x_{1} x_{2}^{-1} x_{1}^{2}>0 x_{1}^{2}>0 x_{1}>0$. Similarly, we need to consider all the other diagrams. Since $x_{1}, x_{2}$ are free generators, we may assume that they fix none of the finite set of "migratory points". Since we have a diagram in which $0 w \neq 0$, we obtain that $w \neq 1$. If $w:=\bigvee_{i \in I} \bigwedge_{j \in J} w_{i, j}$ where $I, J$ are finite index sets and each $w_{i, j}$ is a group word, by examining all the finitely many possible diagrams for this $w$, we see that $w \neq 1$ in $F$ if $0 w \neq 0$ in at least one of these diagrams, and $w=1$ in $F$ if $0 w=0$ in all of these possible diagrams. This solves the word problem for $F$.

These ideas can also be used to prove that the solubility of the word problem is preserved by free products (in the class of $\ell$-groups) and in the proof of Theorem J below; see [30].

\subsection{Amalgamation in Groups and $\ell$-Groups}

To prove the undecidability of various problems in group theory, one uses the free product with amalgamated subgroup. That is, given groups $G_{1}$ and $G_{2}$ with isomorphic subgroups $H_{1}$ and $H_{2}$ (say by $\varphi$ ), one can form a group $L$ generated by $G_{1}$ and $G_{2}$ with embeddings $\psi_{i}: G_{i} \rightarrow L$ $(i=1,2)$ so that $g_{1} \psi_{1}=g_{2} \psi_{2}$ iff there is $h_{1} \in H_{1}$ such that $g_{1}=h_{1}$ and $g_{2}=h_{1} \varphi\left(g_{i} \in G_{i} ; i=1,2\right)$; see, e.g., [51]. In his 1970 Ph.D. thesis under Holland, K. R. Pierce used permutation groups to show that there are $\ell$-groups $G_{1}$ and $G_{2}$ with $\ell$-isomorphic $\ell$-subgroups $H_{1}$ and $H_{2}$ (say by $\varphi$ ) such that there is no $\ell$-group $L$ generated by $G_{1}$ and $G_{2}$ with 
-embeddings $\psi_{i}: G_{i} \rightarrow L(i=1,2)$ so that $h_{1} \psi_{1}=h_{1} \varphi \psi_{2}$ in $L$ for all $h_{1} \in H_{1}$. In contrast, in the same paper [63] Pierce showed that if $H_{1}$ and $H_{2}$ are $\ell$-isomorphic archimedean o-groups, then there is always such an $\ell$-group $L$. This latter proof involved establishing that any $\ell$ group $G$ can be $\ell$-embedded in an $\ell$-group $G^{\#}$ in which any two strictly positive (i.e., $>1$ ) elements are conjugate - since $g>1$ iff $f^{-1} g f>1$, the conjugacy class of a strictly positive element is a subset of the set of all strictly positive elements. To achieve this, he needed to show that if $f, g>1$ in $G$, then there is an $\ell$-group $H:=H(f, g)$ in which $f$ and $g$ are conjugate. This required a tricky transfinite induction using the orbital Wreath product at half the stages. In $G^{\#}$, all strictly negative elements are conjugate since they are the inverses of the strictly positive elements. This left open whether one could find such a $G^{\#}$ in which, additionally, all elements incomparable to the identity could also be made conjugate. This was recently achieved in [7] using an elaboration of Pierce's original construction.

The main obstacle to a free product of right-orderable groups with amalgamated subgroup being right orderable is that a conjugate of the right order on one factor may be incompatible with any right order on the other factor. This is essentially the only obstruction, so one can amalgamate o-groups in the class of right-orderable groups [8]; cf. [66]. To see this one crucially uses ultraproducts of right orders (see [9]). Using "normal" families of right orders on the factors and constructing groups of order-preserving permutations of totally ordered sets (sic), one can provide precise necessary and sufficient conditions for a free product of rightorderable groups with amalgamated subgroup to be right-orderable ( $m u$ tatis mutandis with right-ordered in place of right-orderable) [9]. These ideas extend the non-permutation ideas in [4].

Let $G$ act on $\Omega$ and $g \in G$. The fixed point set of $g$, the set of positive intervals of support of $g$ and the set of negative intervals of support of $g$ form a partition of $\Omega$. Hence for any ultrafilter $\mathcal{U}$ on $\Omega$, exactly one of these three sets belongs to $\mathcal{U}$. Using this and permutation methods mentioned above, one obtains

Theorem D [10] Let $G_{i}$ be a lattice-ordered group with $\ell$-subgroup $H_{i}$ $(i=1,2)$. If $\varphi: H_{1} \cong H_{2}$ is an $\ell$-isomorphism, then the (group) free product of $G_{1}$ and $G_{2}$ (with $H_{1}$ and $H_{2}$ amalgamated by $\varphi$ ) is right- 
orderable. Hence the amalgamation can be achieved in a lattice-orderable group, the embeddings being only group embeddings.

Caution: In general, the lattice-ordering on the overgroup need not be compatible with the original lattice orderings as Pierce's counterexample shows. This obstacle can still prevail when $H_{i}$ is a prime $\ell$-ideal of $G_{i}$ $(i=1,2)$, though in this case the group free product of $G_{1}$ and $G_{2}$ is lattice-orderable (see [10]). Indeed, there are examples (op. cit.) of $H_{1}$ a prime $\ell$-ideal of $G_{1}$ and $H_{2}$ an $\ell$-ideal of $G_{2}$ where there are no amalgamating $\ell$-embeddings of $G_{1}$ and $G_{2}$ into any amalgam $L$ that is lattice-orderable whatever lattice orderings are placed on $G_{1}, G_{2}$ and $L$. This indicates that although there is a very close tie between rightorderable groups and lattice-orderable groups, there are some startling differences.

\subsection{Decision Problems in Right-Orderable Groups and $\ell$-Groups}

As shown in [9], these necessary and sufficient conditions (for a free product of right-orderable groups with amalgamated subgroup to be right orderable) provide the following results where the presentation is as a group.

Theorem E [9] There is a finitely presented right-orderable group with insoluble (group) word problem.

Theorem F ([9], cf. [38]) A finitely generated right-orderable group can be embedded in a finitely presented right-orderable group iff it can be defined by a recursively enumerable set of defining relations (in the language of groups).

Theorem G ([9], cf. [11]) A finitely generated right-orderable group has soluble word problem iff it can be embedded in a simple group which can be embedded in a finitely presented right-orderable group.

Analagous results hold with right-ordered instead of right-orderable (op. cit.).

The ideas in [39] can also be used to provide conditions which imply that two finite sets of strictly positive elements in $A(\mathbb{R})$ (of the same size) 
can be made conjugate by the same conjugator. From this one can obtain the insolubility of the word problem for $\ell$-groups, where an $\ell$-group is said to be finitely presented if it is finitely generated (as an $\ell$-group) and is definable by a finite set of relations (in the language of $\ell$-groups) between the generators.

Theorem $\mathbf{H}$ [33] There is a finitely presented lattice-ordered group with insoluble (group) word problem.

The proof involves forming both a finitely presented $\ell$-group $G(X)$ and an $\ell$-homomorphic image in $A(\mathbb{R})$. Then if $X$ is a recursively enumerable set of natural numbers that is not recursive, there is a set of group words $\left\{w_{n} \mid n \in \mathbb{N}\right\}$ such that from the finite presentation one can obtain $w_{n}=1$ in $G(X)$ if $n \in X$ and, on the other hand, if $n \notin X$, then in the $\ell$-homomorphic image of $G(X)$ in $A(\mathbb{R})$ we have $w_{n} \neq 1$. Hence $w_{n}=1$ in $G(X)$ iff $n \in X$ and $G(X)$ has insoluble group word problem.

The ideas above can be used to prove analogues of the same famous theorems from group theory.

Theorem I ([28], cf. [38]) A finitely generated $\ell$-group can be $\ell$-embedded in a finitely presented $\ell$-group iff it can be defined by a recursively enumerable set of defining relations (in the language of $\ell$-groups).

Theorem J ([29], cf. [11]) A finitely generated $\ell$-group has soluble word problem iff it can be $\ell$-embedded in a simple $\ell$-group which can be $\ell$ embedded in a finitely presented $\ell$-group.

\subsection{First-Order Properties of Automorphism Groups}

R. N. McKenzie showed that if $\Omega$ is any set and $\operatorname{Sym}(\Omega)$ satisfies the same first-order sentences in the language of groups as $\operatorname{Sym}(\mathbb{N})$, then $\Omega$ is countably infinite [58]. M. Giraudet began the study of the first-order theories of $A(\Omega)$ for totally ordered sets $(\Omega, \leq)$ and showed that the lattice structure could be recovered from the group sentences satisfied and vice versa if $A(\Omega)$ is o-2 transitive [48]. Gurevich and Holland showed that if $A(\Omega)$ is transitive and satisfies the same first-order sentences as $A(\mathbb{R})$ in the language of $\ell$-groups, then there is an order-preserving bijection between $(\Omega, \leq)$ and $(\mathbb{R}, \leq)$ (see [36]). The ideas are pictorial and similar to those begun in [39]. For further results, see [31], Chapter 2. 


\subsection{Commutators and the Bergman Property}

Having every element a commutator is a very interesting property (see [62], [5] and especially [50] where it is shown that every element of any finite simple group is a commutator). Also, recall that each o-2 transitive group $A(\Omega)$ is divisible. These two results can be unified and extended as follows. Given a group $G$, a word $w=w\left(x_{1}, \ldots, x_{n}\right) \in F$ of the free group is called universal in $G$, if for each $g \in G$ there are $g_{1}, \ldots, g_{n} \in G$ such that $g=w\left(g_{1}, \ldots, g_{n}\right)$. As noted in Subsection 2.1, the commutator word $\left[x_{1}, x_{2}\right]$ and all powers $x^{n}$ are universal in each o-2 transitive group $A(\Omega)$. Adeleke and Holland [1] showed that in fact each non-trivial word $w \neq 1$ is universal in each o-2 transitive group $A(\Omega)$.

We say that a group $G$ satisfies the Bergman property if whenever $J$ is a set of generators of $G$, there is a positive integer $n=n(J)$ such that each element of $G$ is a product of at most $n$ members of $J \cup J^{-1}$. Recently, Bergman [5] proved the remarkable result that the full symmetric group $\operatorname{Sym}(\Omega)$ of all permutations of any infinite set $\Omega$ has the Bergman property. The methods used were similar to those of Macpherson and Neumann [52] who showed that $\operatorname{Sym}(\Omega)$ has uncountable cofinality; i.e., cannot be written as the union of a countable tower of proper subgroups; see the survey [65] for further results.

In our context, Gourion [35] showed that $A(\mathbb{Q})$ has uncountable cofinality. Using a uniform argument, it was shown in [16] that any o-2 transitive group $A(\Omega)$ has the Bergman property and uncountable cofinality. As a consequence of the results in $[18,16]$, any o-2 transitive automorphism group $A(T)$ of a countable tree $(T, \leq)$ has the Bergman property and uncountable cofinality [23]. In [20], these properties were also derived for further infinite permutation groups, including groups of measure-preserving or ergodic transformations of $\mathbb{R}$.

\subsection{Lattice-ordered groups with a unit}

Let $G$ be an $\ell$-group and $u \in G$ be strictly positive. We say that $u$ is a strong order unit (or unit, for short) if for each $g \in G$, there is $n=n(g) \in \mathbb{Z}_{+}$such that $u^{-n}<g<u^{n}$. For example, if $G$ is periodic with period $z$ and $z \in G$, then $z$ is a unit of $G$. And if $G$ is a torsion-free nilpotent group, then $G$ can be made into an o-group with unit $u$ iff $u \in G \backslash \gamma_{2}(G)$. 
D. Mundici has exhibited an explicit categorical equivalence between multi-valued algebras (associated with multi-valued logics) and abelian $\ell$-groups with a unit [59], and Dvurecenskij extended the result to the non-commutative case [24]. This interplay allows one to study varieties of not-necessarily-abelian multi-valued algebras by studying equational classes of $\ell$-groups with unit. That is, in the language of $\ell$-groups with an added constant symbol $u$. Throughout this subsection, we will assume that $u$ is a unit. Since this cannot be expressed first order, we merely get equational classes in this language, not varieties. Nonetheless, the techniques of [39] are available and open up this new area of research.

Equational classes of $\ell$-groups with unit were introduced in [25]. If $(G, \Omega)$ is an $\ell$-permutation group and $u$ is a unit of $G$, then there is an o-primitive component of $G$ on which $u$ acts non-trivially. This is called the top o-primitive component of $G$. It is an $\ell$-homomorphic image of a sublattice subgroup of $G$ and has the image of $u$ as a unit. The key result is that if $w$ is an $\ell$-group word in $u$ and the generators of $G$, then $w=1$ in the top component iff $w^{\prime} \leq u$ for every product of conjugates $w^{\prime}$ of $w$. Hence if $\mathcal{V}$ is an equational class of $\ell$-groups with unit $u$, then the collection of all $\ell$-groups with unit $u$ whose top components lie in $\mathcal{V}$ is an equational class of $\ell$-groups with unit $u$; it is called a top equational class. M. R. Darnel has shown that each top equational class includes all normal-valued $\ell$-groups with unit (personal communication). Whereas any non-abelian $\ell$-group with a faithful primitive representation generates the variety of all $\ell$-groups (Theorem $\mathrm{C}$ ), this is no longer true for periodic o-primitive $\ell$-permutation groups with a unit; indeed, the equational class generated by any periodic o-primitive $\ell$-permutation group with a unit is never the equational class of all $\ell$-groups with unit. Furthermore, there are $2^{\aleph_{0}}$ distinct top equational classes generated by periodic o-primitive $\ell$-permutation groups (see [44]). This has many interesting ramifications.

Even the normal subgroup picture is more diverse in this setting. Recall that $A(\mathbb{R})$ has only three proper normal subgroups, $B(\mathbb{R}), L(\mathbb{R})$ and $R(\mathbb{R}$ ). Let $u$ be translation by 1 (so $u: \alpha \mapsto \alpha+1$ ) and let $G$ be the convex sublattice subgroup of $A(\mathbb{R})$ generated by $u$; so $G$ has unit $u$ and $G:=\left\{g \in A(\mathbb{R}) \mid\left(\exists n \in \mathbb{Z}_{+}\right)(\forall \alpha \in \mathbb{R})(|\alpha g-0 g| \leq n)\right\}$. There are some surprises. Recall that an ideal of $P(\mathbb{Z})$ is a non-empty subset of $P(\mathbb{Z})$ closed under taking unions and subsets. We call an ideal $\mathcal{K}$ of 
$P(\mathbb{Z})$ normal, if for any $K \in \mathcal{K}$ and $n \in \mathbb{N}$ the set $K^{<n>}=\{m \in \mathbb{Z} \mid$ $(\exists k \in K)(|m-k| \leq n)\}$ also belongs to $\mathcal{K}$. By [17], the normal subgroup lattice of $G$ is isomorphic to the lattice of all normal ideals of $P(\mathbb{Z})$. As a consequence, $G$ has chains of normal subgroups isomorphic to $(\mathbb{R}, \leq)$ and antichains of normal subgroups of size $2^{2^{\aleph_{0}}}$.

\subsection{Outer automorphism groups}

As is well-known, each automorphism of $A(\mathbb{Q})$ or of $A(\mathbb{R})$ arises via conjugation by a group element or an anti-isomorphism of $(\mathbb{R}, \leq)$. Hence the outer automorphism group $\operatorname{Out}(A(\Omega))=\operatorname{Aut}(A(\Omega)) / \operatorname{Inn}(A(\Omega)) \cong \mathbb{Z} / 2 \mathbb{Z}$ for $\Omega=\mathbb{Q}$ and $\Omega=\mathbb{R}$. Holland [42] and Weinberg [68] constructed ordered sets $(\Omega, \leq)$ with o-2 transitive $A(\Omega)$ for which $\operatorname{Out}(A(\Omega))$ is trivial. Solving a problem which had been open for some time, Holland [42] constructed such an order $(\Omega, \leq)$ with $\operatorname{Out}(A(\Omega)) \cong V_{4}$, the Klein fourgroup. More recently, it was shown in [53] that any group $G$ arises as outer automorphism group $G \cong \operatorname{Out}(H)$ of some group $H$. The question arises which groups arise as $\operatorname{Out}(A(\Omega))$ for some o-2 transitive group $A(\Omega)$.

Theorem K [22] Let $G$ be any group. Then there exists a totally ordered set $(\Omega, \leq)$ such that $A(\Omega)$ is o-2 transitive and $G \cong \operatorname{Out}(A(\Omega))$.

The proof proceeds by encoding $G$ via stationary subsets within some ordered set, and then constructing $\Omega$ 'around it', preserving existing suprema and infima as indicated at the end of Subsection 2.1.

Schreier's conjecture asserts that the outer automorphism group of each finite simple group is soluble (which is true, by the classification of finite simple groups). Without the word 'finite', this changes drastically.

Theorem L [15] Let $G$ be any group. Then $G \cong$ Out $(H)$ for some simple group $H$. Moreover, $H$ may be chosen as the automorphism group $H=A u t(C, R)$ for some ternary relational structure $(C, R)$.

This group-theoretic result appears as a consequence of the preceding $\ell$-permutation group methods as follows. By Theorem $\mathrm{K}$, we have $G \cong$ $\operatorname{Out}(A(\Omega))$ for some totally ordered set $(\Omega, \leq)$ with $A(\Omega)$ o-2 transitive. As observed in Subsection 2.2, $A(\Omega)$ is not simple. We may construct $(\Omega, \leq)$ so that it contains a countable subset with no upper or lower 
bound in $\Omega$. Then there are $\alpha \in \Omega$ and $z \in A(\Omega)$ such that $\left\{\alpha z^{n} \mid n \in \mathbb{Z}\right\}$ is unbounded above and below in $\Omega$; thus $\left(C_{A(\Omega)}(z), \Omega\right)$ is periodic with period $z$. We put $C=[\alpha, \alpha z)$, and let $R$ comprise all triples $(\beta, \gamma, \delta) \in C^{3}$ such that $\beta<\gamma<\delta$ or $\gamma<\delta<\beta$ or $\delta<\beta<\gamma$; hence $R$ is a "circular ordering' of $C$. Then $\operatorname{Out}(A(\Omega))$ is isomorphic to the group of those permutations of the $A(\Omega)$-orbits in $\bar{\Omega}$ which are induced by elements of $A(\bar{\Omega})$, and this correspondence transfers to $\operatorname{Out}(\operatorname{Aut}(C, R))$ by a recent analysis in [57]. Hence, $G \cong \operatorname{Out}(A(\Omega)) \cong \operatorname{Out}(\operatorname{Aut}(C, R))$. Moreover, $\operatorname{Aut}(C, R) \cong C_{A(\Omega)}(z) /\langle z\rangle$, which is simple by [47].

\section{Conclusion}

By examining both Theorem A and its proof, we have attempted to provide the necessary background to appreciate the many important consequences that were subsequently obtained. By choosing applications in diverse areas, we hope to have adequately illustrated the importance of Holland's original theorem.

\section{References}

[1] S. A. Adeleke and W. C. Holland, Representation of order automorphisms by words, Forum Mathematicum 6 (1994), 315-321.

[2] S. A. Adeleke and P. M. Neumann, Relations related to betweenness: their structure and automorphisms, Memoirs American Math. Soc. 131 (1998), no. 623.

[3] R. N. Ball and M. Droste, Normal subgroups of doubly transitive automorphism groups of chains, Transactions American Math. Soc. 290 (1985), 647-664.

[4] G. M. Bergman, Ordering coproducts of groups and semigroups, J. Algebra 133 (1990), 313-339.

[5] G. M. Bergman, Generating infinite symmetric groups, Bull. London Math. Soc. 38 (2006) 429-440. 
[6] V. V. Bludov, Completion of a linearly ordered group, Algebra and Logic 44 (2005), 370-380.

[7] V. V. Bludov and A. M. W. Glass, Conjugacy in lattice-ordered and right orderable groups, J. Group Theory 11 (2008), 623 - 633.

[8] V. V. Bludov and A. M. W. Glass, On free products of right ordered groups with amalgamated subgroups, Math. Proc. Cambridge Philosophical Soc. 146 (2009), 591-601.

[9] V. V. Bludov and A. M. W. Glass, Word problems, embeddings, and free products of right-ordered groups with amalgamated subgroup, Proc. London Math. Soc. doi: 10.1112/plms/pdp008.

[10] V. V. Bludov and A. M. W. Glass, Right orders and amalgamation for lattice-ordered groups, Math. Slovaca (to appear).

[11] W. W. Boone and G. Higman, An algebraic characterization of the solvability of the word problem, J. Australian Math. Soc. 18 (1974), 41-53.

[12] P. M. Cohn, Groups of order automorphisms of ordered sets, Mathematika 4 (1957), 41-50.

[13] M. Droste, The normal subgroup lattice of 2-transitive automorphism groups of linearly ordered sets, Order 2 (1985), 291-319.

[14] M. Droste, Complete embeddings of linear orderings and embeddings of lattice-ordered groups, Israel J. Math. 56 (1986), 315-334.

[15] M. Droste, M. Giraudet, and R. Göbel, All groups are outer automorphism groups of simple groups, J. London Math. Soc. (2) 64 (2001), 565-575.

[16] M. Droste and W. C. Holland Generating automorphism groups of chains, Forum Mathematicum 17 (2005), 699-710.

[17] M. Droste and W. C. Holland, Normal subgroups of $\mathrm{B}_{\mathrm{u}}$ Aut $(\Omega)$, Appl. Categor. Structures 15 (2007), 153-162.

[18] M. Droste, W. C. Holland, and H. D. Macpherson, Automorphism groups of infinite semilinear orders (I) and (II), Proc. London Math. Soc. (3) 58 (1989), 454-478 and 479-494. 
[19] M. Droste, W. C. Holland, and H. D. Macpherson, Automorphism groups of homogeneous semilinear orders: Normal subgroups and commutators, Canadian J. Math. 43 (1991), 721-737.

[20] M. Droste, W. C. Holland, and G. Ulbrich, On full groups of measure-preserving and ergodic transformations with uncountable cofinalities, Bull. London Math. Soc. 40 (2008), 463-472.

[21] M. Droste and S. Shelah, A construction of all normal subgroup lattices of 2-transitive automorphism groups of linearly ordered sets, Israel J. Math. 51 (1985), 223-261.

[22] M. Droste and S. Shelah, Outer automorphism groups of ordered permutation groups, Forum Mathematicum 14 (2002), 605-621.

[23] M. Droste and J. K. Truss, Uncountable cofinalities of automorphism groups of linear and partial orders, Algebra Universalis (to appear).

[24] A. Dvurecenskij, Pseudo-MV algebras are intervals of unital $\ell$ groups, J. Australian Math. Soc. 72 (2002), 427-445.

[25] A. Dvurecenskij and W. C. Holland, Top varieties of generalized MV-algebras and unital lattice-ordered groups, Comm. Algebra 35 (2007), 3370-3390.

[26] A. M. W. Glass, $\ell$-simple lattice-ordered groups, Proc. Edinburgh Math. Soc. 19 (1974), 133-138.

[27] A. M. W. Glass, Free products of lattice-ordered groups, Proc. American Math. Soc. 101 (1987), 11-16.

[28] A. M. W. Glass, Sublattice subgroups of finitely presented latticeordered groups, J. Algebra 301 (2006), 509-530.

[29] A. M. W. Glass, Finitely generated lattice-ordered groups with soluble word problem, J. Group Theory 11 (2008), 1-21.

[30] A. M. W. Glass, Free products and Higman-Neumann-Neumann type extensions of lattice-ordered groups, in Ischia Group Theory 2006, (ed. T. Hawkes et. al), World Sci. Pub. Co., Singapore, 2007, pp. $147-165$. 
[31] A. M. W. Glass, Ordered Permutation Groups, London Math. Soc. Lecture Notes Series 55, Cambridge University Press, Cambridge, 1981.

[32] A. M. W. Glass, Partially Ordered Groups, Series in Algebra 7, World Scientific Pub. Co., Singapore, 1999.

[33] A. M. W. Glass and Y. Gurevich, The word problem for latticeordered groups, Transactions American Math. Soc. 280 (1983), 127138.

[34] A. M. W. Glass, W. C. Holland and S. H. McCleary, The structure of $\ell$-group varieties, Algebra Universalis 10 (1980), 1-20.

[35] C. Gourion, À propos du groupe des automorphismes de $(\mathbb{Q}, \leq)$, C. R. Acad. Sci. Paris Sér. I Math. 315 (1992), 1329-1331.

[36] Y. Gurevich and W. C. Holland, Recognizing the real line, Transactions of the American Math. Soc. 265 (1981), 527-534.

[37] G. Higman, On infinite simple groups, Pub. Math. Debrecen 3 (1954), 221-226.

[38] G. Higman, Subgroups of finitely presented groups, Proc. Royal Soc. London Ser. A 262 (1961), 455-475.

[39] W. C. Holland, The lattice-ordered group of automorphisms of an ordered set, Michigan Math. J. 10 (1963), 399-408.

[40] W. C. Holland, Transitive lattice-ordered permutation groups, Math. Z. 87 (1965), 420-433.

[41] W. C. Holland, The characterization of generalized wreath products, J. Algebra 13 (1969), 155-172.

[42] W. C. Holland Outer automorphisms of ordered permutation groups, Proc. Edinburgh Math. Soc. 19 (1974), 331-344.

[43] W. C. Holland, The largest proper variety of lattice-ordered groups, Proc. American Math. Soc. bf 57 (1976), 25-28. 
[44] W. C. Holland, Continuum many top varieties of GMV-algebras and unital lattice-ordered groups, Algebra Universalis "Conrad Volume" (to appear).

[45] W. C. Holland and S. H. McCleary, Wreath products of ordered permutation groups, Pacific J. Math. 31 (1969), 703-716.

[46] W. C. Holland and S. H. McCleary, The word problem for free latticeordered groups, Houston J. Math. 5 (1979), 99-105.

[47] S. P. Hurd, Normal subgroups of periodically o-primitive ordered permutation groups, Houston J. Math. 15 (1989), 85-98.

[48] M. Jambu-Giraudet, Bi-interpretable groups and lattices, Transactions American Math. Soc. 278 (1983), 253-269.

[49] V. M. Kopytov, Free lattice-ordered groups, Algebra and Logic 18 (1979), 259-270.

[50] M. W. Liebeck, E. O'Brien, A. Shalev, P. H. Tiep, The Ore Conjecture (submitted).

[51] R. C. Lyndon and P. E. Schupp, Combinatorial Group Theory, Ergebnisse der Math. und ihrer Grenzgebiete 89, Springer-Verlag, Heidelberg, 1977.

[52] H. D. Macpherson and P. M. Neumann, Subgroups of infinite permutation groups, J. London Math. Soc. (2) 42 (1990), 64-84.

[53] T. Matumoto Any group can be represented by an outer automorphism group, Hiroshima Math. J. 19 (1989), 209-219.

[54] S. H. McCleary, o-primitive ordered permutation groups I and II, Pacific J. Math. 40 (1972), 349-372; 49 (1973), 431-443.

[55] S. H. McCleary, The structure of intransitive ordered permutation groups, Algebra Universalis 6 (1976), 229-255.

[56] S. H. McCleary, Free lattice-ordered groups represented as o-2 transitive $\ell$-permutation groups, Transactions American Math. Soc. 290 (1985), 69-100. 
[57] S. H. McCleary and M. Rubin, Locally moving groups and the reconstruction problem for chains and circles, 1995; http://arxiv.org/abs/math/0510122.

[58] R. McKenzie, On elementary types of symmetric groups, Algebra Universalis 1 (1971), 13-20.

[59] D. Mundici, Interpretation of $A F C^{*}$-algebras in Lukasiewicz sentential calculus, J. Functional Analysis 65 (1986), 15-63.

[60] A. Navas and C. Rivas, A new characterization of Conrad's property for group orderings, with applications (with an appendix by A. Clay), arxiv:math/0901.0880.

[61] T. Ohkuma, Sur quelques ensembles ordonnés linéairement, Fund. Math. 43 (1955), 326-337.

[62] O. Ore, Some remarks on commutators, Proc. American Math. Soc. 2 (1951), 307-314.

[63] K. R. Pierce, Amalgamations of lattice-ordered groups, Transactions American Math. Soc. 172 (1972), 249-260.

[64] J. A. Read, Wreath products of non-overlapping lattice-ordered groups, Canadian Math. Bull. 17 (1975), 713-722.

[65] S. Thomas, Cofinalities of infinite permutation groups, in: Advances in Algebra and Model Theory (eds. M. Droste and R. Göbel), Gordon Breach, Amsterdam, 1997, pp. 101-120.

[66] A. A. Vinogradov, On the free product of ordered groups, Math. Sbornik 25 (1949), 163-168 (in Russian).

[67] E. C. Weinberg, Embedding in a divisible lattice-ordered group, J. London Math. Soc. 42 (1967), 504-506.

[68] E. C. Weinberg, Automorphism groups of minimal $\eta_{\alpha}$-sets, in: Ordered Groups (eds. J.E. Smith, G.O. Kenny, and R.N. Ball), Lecture Notes in Pure and Applied Math. 62, Marcel Dekker, New York, 1980, pp. 71-79. 
[69] S. Wolfenstein, Valeurs normales dans un groupe réticulé, Acad. Naz. dei Lincei 44 (1968), 337-342.

Authors' addresses:

V. V. Bludov:

Department of Mathematics, Physics, and Informatics, Irkutsk State Teachers Training University,

Irkutsk 664011,

Russia

vasily-bludov@yandex.ru

Manfred Droste:

Institut für Informatik,

Universität Leipzig,

D-04109 Leipzig,

Germany

droste@informatik.uni-leipzig.de

A. M. W. Glass:

Department of Pure Mathematics and Mathematical Statistics, Centre for Mathematical Sciences,

Wilberforce Rd.,

Cambridge CB3 0WB,

England

amwg@dpmms.cam.ac.uk 